\title{
Assessing the anomalous superdiffusive heat transport in a single one-dimensional PEDOT chain
}

\author{
Alessandro Crnjar, Claudio Melis, and Luciano Colombo* \\ Department of Physics, University of Cagliari, Cittadella Universitaria, 09042 Monserrato, Italy
}

(Received 6 September 2017; published 29 January 2018)

\begin{abstract}
We present a computational investigation on heat transport in a single polymer chain of poly-3,4ethylenedioxythiophene (PEDOT). By applying equilibrium and nonequilibrium molecular dynamics simulations to evaluate the thermal conductivity, as well as by investigating how the polymer chain approaches equilibrium upon a local thermal excitation, we provide a robust picture assessing the anomalous superdiffusive (i.e., intermediate between ballistic and diffusive) character of its thermal transport. This assessment is provided by the present simulations showing that three scaling laws with unlike physical meaning and characterizing the thermal energy transport in one-dimensional systems indeed occur in the very same polymer chain with consistent critical exponents. In order to disentangle the effect of dimensionality, we perform a systematic comparison of transport features for a single one-dimensional (1D) PEDOT chain and a three-dimensional (3D) PEDOT crystal. Present simulations suggest that by increasing the dimensionality, the anomalous regime is completely removed as due to the occurrence of strong interchains anharmonic interactions. Finally, we prove that thermal transport in isolated single PEDOT chains belongs to a novel universality class of superdiffusion characterized by a critical exponent $\beta=1 / 2$.
\end{abstract}

DOI: 10.1103/PhysRevMaterials.2.015603

\section{INTRODUCTION}

Nonequilibrium diffusive heat transport is macroscopically described by the Fourier law, linking the heat current vector $\vec{J}$ to the applied thermal gradient $\vec{\nabla} T$ through a phenomenological constitutive equation as $\vec{J}=-\kappa \vec{\nabla} T$. The thermal conductivity $\kappa$ is an intensive material property described by a scalar constant in homogeneous and isotropic media. For insulating systems the microscopic heat carriers are lattice vibrations and the Fourier law is obeyed when the system is found close to global equilibrium (i.e., $\vec{\nabla} T$ is small enough) $[1,2]$. This state of affairs breaks down in low-dimensional systems: many theoretical as well as experimental works reported an anomalous (i.e., non-Fourier) heat conduction regime in quasi-1D objects, highlighting a strong dependence of thermal conductivity on the system size. In particular, the nonintensive character of $\kappa$ has been experimentally observed in 1D-like carbon and boron-nitride nanotubes [3,4] as well as in SiGe nanowires [5], while a similarly characterized anomalous heat conduction has been theoretically shown to occur in 1D Fermi-Pasta-Ulam [6] and diatomic Toda lattices [7]. Atomistic simulations as well predict anomalous heat conduction in different 1D-like materials, such as carbon nanotubes [8,9], silicon nanowires [10], and polymer chains [11-14].

The search for anomalous features in heat transport is actively ongoing since the physical background is partly unclear. For instance, although the reduced dimensionality is recognized to be a necessary condition for anomalous transport, some theoretical works claimed that it is not the only feature establishing such a regime $[15,16]$ : normal heat conduction (described by the ordinary Fourier law with an

*luciano.colombo@dsf.unica.it intensive $\kappa$ ) has been in fact observed for 1D lattices interacting via Lennard-Jones, Morse, and Coulomb-like potentials [16], thus making the issue still a matter of discussion.

Recently, a general picture on heat transport in 1D systems has been presented [17], where it is discussed that anomalous transport, if occurring, is highlighted by scaling laws, both in time and space, characterized by interlaced critical exponents. The synopsis of possible behaviors is as follows:

(1) The thermal conductivity $\kappa$ could depend on the system length $L_{x}$ as

$$
\kappa \propto L_{x}^{\beta}
$$

Hereafter $x$ will be assumed as the direction of heat transport. Depending on the $\beta$ value the following taxonomy holds [17,18]: $\beta=1$ corresponds to a ballistic regime, where the thermal conductivity is proportional to the length of the system; $0<\beta<1$ corresponds to a superdiffusive regime characterized by a divergent thermal conductivity and a competition between ballistic and normal transport; $\beta=0$ corresponds to a normal diffusion regime in which the system follows the Fourier law; finally, $\beta<0$ corresponds to a subdiffusive regime with a vanishing thermal conductivity in the thermodynamic limit. Among the systems showing superdiffusive regime, most theoretical works agree that the actual $\beta$ value should be lower than $0.4[17,19,20]$. However the possible existence of a different universality class of superdiffusion with $\beta=1 / 2$ is largely debated [21]. It has been proposed that such a $\beta$ value should occur only for even interaction potentials, i.e., symmetric with respect to the equilibrium position. We emphasize that, up to now, the existence of this regime has been only addressed by considering "ideal" relatively simple model potentials (like the Fermi-Pasta-Ulam one), while neither experiments nor atomistic simulations using more realistic model potentials 
have been exploited to prove the existence of such a novel universality class of transport.

(2) The time decay of the heat current autocorrelation function $\langle J(t) J(0)\rangle$ could result to be not integrable, yielding to a divergent value of the Green-Kubo integral and, therefore, of the corresponding $\kappa$ :

$$
\kappa(t)=\frac{1}{V K_{B} T^{2}} \int_{0}^{t}\langle J(t) J(0)\rangle d t,
$$

where $V$ is the volume of the system, $K_{B}$ is the Boltzmann constant, $T$ is the temperature of the system, $t$ is the correlation time, and the angular brackets denote ensemble averages. In particular, it has been shown $[17,20]$ that in an anomalous regime the heat current autocorrelation function displays a nonintegrable power law decay:

$$
\langle J(t) J(0)\rangle \propto t^{-(1-\delta)},
$$

with $0 \leqslant \delta<1$ for $t \longrightarrow \infty$. Accordingly, the thermal conductivity $k(t)$ should diverge in time as

$$
\kappa(t) \propto t^{\delta},
$$

with $\delta=\beta$.

(3) The broadening $\sigma$ of a local energy perturbation could evolve in time as $[9,10]$

$$
\sigma^{2}(t)=\sum_{i} \frac{\left[E_{i}(t)-E_{0}\right]\left(\vec{r}_{i}(t)-\vec{r}_{0}\right)^{2}}{\sum_{i}\left[E_{i}(t)-E_{0}\right]},
$$

where $E_{i}(t)$ and $\vec{r}_{i}(t)$ are the energy and the position of atom $i$ at time $t$, while $\overrightarrow{r_{0}}$ and $E_{0}$ are, respectively, the position of an energy pulse at $t=0$ and the energy per atom for the unperturbed equilibrium configuration. In the $1 \mathrm{D}$ case, the time evolution of $\sigma$ follows the equation [17]

$$
\sigma^{2}(t) \propto t^{\alpha},
$$

where $\beta=\alpha-1$. This relation describes the physical connection between energy diffusion and thermal conductivity. Thus, in normal diffusion $\alpha=1$ and correspondingly $\beta=0$, which means that the thermal conductivity is a size-independent constant: thermal transport is diffusive and the Fourier law is valid. In the opposite case, i.e., in the ballistic regime, we have $\alpha=2$, and thus $\beta=1$, namely the thermal conductivity of the system is infinite in the thermodynamic limit.

Although the outlined theoretical picture is general and robust, we remark that we still miss a validation (either experimental or based on atomistic simulations) that the above threefold set of scaling laws is in fact occurring on the very same system in the specific case of non-Fourier transport. As a matter of fact, basically all reports on anomalous heat transfer have been so far based on the observation of just one among the three signatures provided by Eqs. (1), (4), and (6), respectively. Moreover, as mentioned above, none of the previous experimental or computational works identified the occurrence of a novel universality class within the superdiffusive regime (corresponding to $\beta=1 / 2$ ), whose existence have been theoretically predicted.

This motivates our present investigation, which is addressed to assess possible anomalous heat transport in realistic quasi1D systems. More specifically, our goal is threefold, namely (i) to provide evidence of the consistent occurrence of different scaling behaviours (i.e., scaling laws must hold and the corresponding critical exponents must fulfill the predicted mutual relationships), (ii) to investigate the role of dimensionality in determining the actual transport regime, and (iii) investigate the possible existence of a novel universality class within the superdiffusive thermal transport regime.

Our investigation is based on atomistic simulations (which allow us to set up thermal situations precisely matching the constitutive hypotheses underlying the above scaling laws) and addressed single polymer chains (which represent a realistic $1 \mathrm{D}$ system). We believe this achievement is helpful in improving our fundamental understanding of heat transport in lowdimensional systems as well as valuable for practical applications of polymers in current nanotechnologies. We focused in particular on poly(3,4-ethylenedioxythiophene) (PEDOT), a prototypical conjugated polymer extensively investigated in literature due to its tunable high electrical conductivity, air stability, and transparency [22]. PEDOT is currently used in its doped state as the hole injection/transport layer in organic (opto)electronic devices. Moreover, the combination of high electrical conductivity and low thermal conductivity makes doped PEDOT a very promising candidate for thermoelectric applications [23-25].

We developed a series of proof-of-concept simulations based on equilibrium (E) and nonequilibrium (NE) classical molecular dynamics (MD). In particular, we perform approach to equilibrium molecular dynamics (AEMD) simulations to investigate the thermal conductivity dependence on the chain length as expressed in Eq. (1), EMD simulations to investigate the time dependence of the Green-Kubo integral as expressed in Eq. (4), and NEMD simulations to investigate the broadening of an energy perturbation during the approach to equilibrium as expressed in Eq. (6). AEMD, EMD, and NEMD simulations are outlined in Fig. 1. We considered single PEDOT chains with a maximum length of $\sim 7.5 \mu \mathrm{m}$. Moreover, we performed a systematic comparison between heat transport in 1D single PEDOT chains vs $3 \mathrm{D}$ crystalline PEDOT samples in order to better focus on the role played by dimensionality. The paper is organized in a section dedicated to the Methods (Sec. II), where the interaction potential and the simulation protocols are carefully described, and in a section where results are presented and discussed (Sec. III). General conclusions are eventually drawn.

\section{METHODS}

All MD simulations have been performed by using the LAMMPS code [26] and the AMBER force field [27]:

$$
\begin{aligned}
E_{\text {total }}= & \sum_{\text {bonds }} K_{r}\left(r-r_{\mathrm{eq}}\right)^{2}+\sum_{\text {angles }} K_{\theta}\left(\theta-\theta_{\mathrm{eq}}\right)^{2} \\
& +\sum_{\text {dihedrals }} \frac{V_{n}}{2}[1+\cos (n \phi)-\gamma]+\sum_{i<j} \frac{q_{i} q_{j}}{r_{i j}} \\
& +\sum_{i<j} 4 \epsilon_{i j}\left[\left(\frac{\sigma_{i j}}{r_{i j}}\right)^{12}-\left(\frac{\sigma_{i j}}{r_{i j}}\right)^{6}\right],
\end{aligned}
$$

including three bonding terms (respectively involving bonds, angles, and dihedrals) and two nonbonding Coulomb and van 


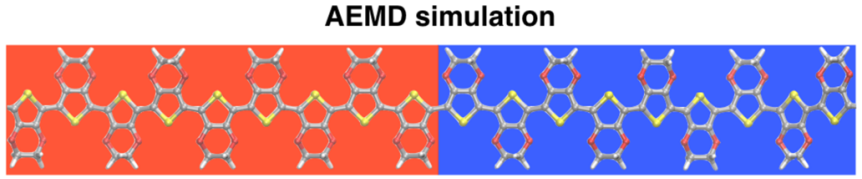

Green-Kubo simulation
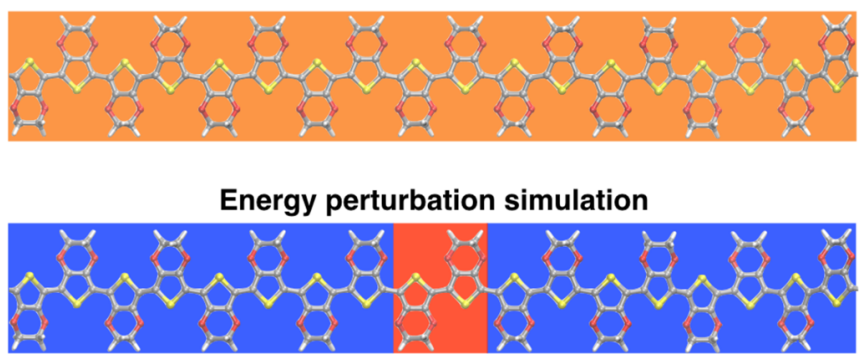

FIG. 1. Schematic layout for the simulation setup of approach to equilibrium MD simulations (top), Green-Kubo equilibrium MD simulations (middle), and time evolution of a local energy perturbation (bottom) performed in this work. The color code represents the initial temperature distribution: blue, orange, and red color corresponds to lower than, equal to, and higher than room temperature, respectively. Over the PEDOT chain (represented by stick-and-ball) it has been initially set as a steplike profile in AEMD, a uniform distribution in Green-Kubo calculations, and a local thermal excitation in the last case.

der Waals (vdW) contributions, respectively, given by the last two sums in Eq. (7) The torsion term can also include so-called "improper" torsions, where the four atoms defining the angle are not all connected by covalent bonds. The parameters occurring in the bonding terms (i.e., $K_{r}, r_{\text {eq }}, K_{\theta}, \theta_{\text {eq }}, V_{n}, n$, and $\gamma$ ) and in the $\operatorname{VdW}$ term (i.e., $\sigma_{i j}$ and $\epsilon_{i j}$ ) have been taken from the GAFF database [28]. Atomic partial charges are estimated with the restrained electrostatic potential (RESP) method [29] using an HF/6-31G* QM calculation to generate the electrostatic potential. The AMBER represents a realistic force field which is currently used to study many properties of different organic materials such as polymers [30], proteins [31], as well as single molecules [32].

The velocity-Verlet algorithm with a time step of 0.5 fs was used to solve the equations of motion. A particle-particle particle-mesh solver is used for the long-range electrostatic forces, and the vdW interactions are cut off at $0.1 \mathrm{~nm}$. The Nosé-Hoover thermostat with corresponding relaxation time equal to $10 \mathrm{fs}$ controlled on-the-fly the temperature. Periodic boundary conditions were imposed along the chain direction $x$, i.e., the polymer chain length and the simulation cell length $L_{x}$ coincide.

The $\kappa$ vs $L_{x}$ dependence was investigated by means of the AEMD method $[33,34]$, in which the system is studied in a transient regime coupling an initial nonequilibrium condition to a final equilibrium state. By considering the heat equation in one dimension:

$$
\frac{\partial T}{\partial t}=\bar{\kappa} \frac{\partial^{2} T}{\partial x^{2}}
$$

(where $\bar{\kappa}=\kappa / \rho c_{v}$ is the thermal diffusivity of the system with mass density $\rho$ and specific heat $c_{v}$ ), we impose an initial steplike temperature profile along the simulation cell. In particular,

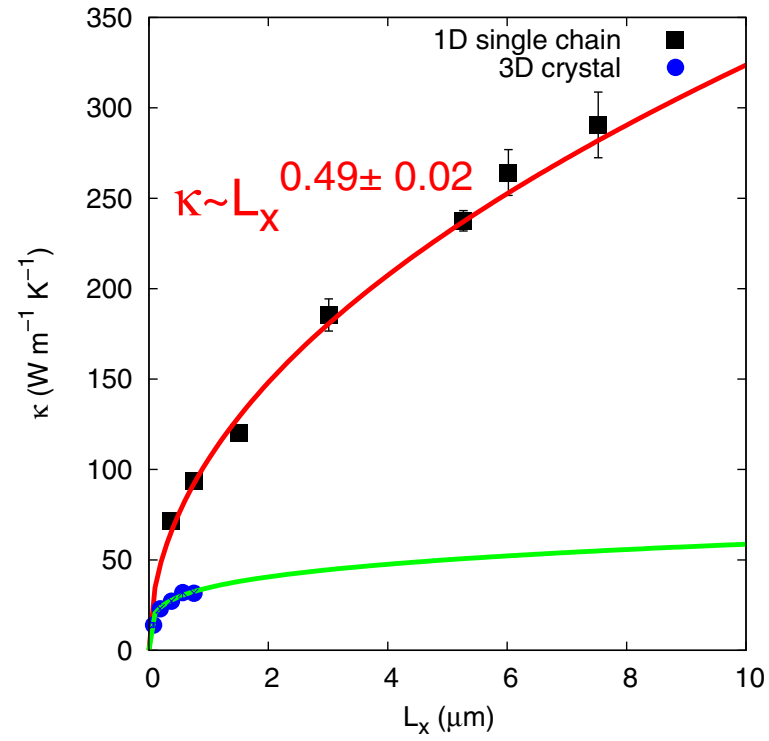

FIG. 2. Thermal conductivity as a function of the system length $L_{x}$ for a 1D PEDOT chain (black squares) and a 3D PEDOT crystal (blue dots). The red (green) line corresponds to Eq. (1).

in the semicell $0 \leqslant x<L_{x} / 2$ the initial temperature is $T_{0}(x)=$ $T_{1}$, while in the semicell $L_{x} / 2 \leqslant x<L_{x}$ it is $T_{0}(x)=T_{2}$, with $T_{1}>T_{2}$. The configuration is then aged by microcanonical molecular dynamics, and the initial steplike temperature profile is smoothed out toward a uniform temperature.

By considering the solution of Eq. (8), we define the average temperature in the two regions $\left\langle T_{1}\right\rangle,\left\langle T_{2}\right\rangle$ and, therefore, the time-dependent average temperature difference

$$
\Delta T(t)=\left\langle T_{1}\right\rangle-\left\langle T_{2}\right\rangle=\sum_{n=1}^{\infty} C_{n} e^{-\alpha_{n}^{2} \bar{\kappa} t},
$$

where $\alpha_{n}=2 \pi n / L_{x}$ and the coefficients $C_{n}=8\left(T_{1}-\right.$ $\left.T_{2}\right)\left[\cos \left(\alpha_{n} L_{x} / 2\right)-1\right]^{2} / \alpha_{n}^{2} L_{x}^{2}$ are determined by the initial conditions. The $\Delta T(t)$ temperature difference, computed during the MD simulation, can be fitted by Eq. (9) in order to determine both the thermal diffusivity $\bar{\kappa}$ and conductivity $\kappa$ through $\bar{\kappa}=\kappa / \rho c_{v}$.

We considered single PEDOT chains with a total number of monomers $500 \leqslant N_{m} \leqslant 10000$ corresponding to $0.376 \leqslant$ $L_{x} \leqslant 7.526 \mu \mathrm{m}$. In order to estimate the system mass density we need to provide its volume $V_{\text {chain. }}$. To this aim $V_{\text {chain }}$ is defined as the length of the simulation cell $L_{x}$ multiplied for the cross-sectional area ( $36 \AA^{2}$ ) of crystalline PEDOT unit cell obtained in Ref. [25].

\section{RESULTS AND DISCUSSION}

Figure 2 shows the thermal conductivity as a function of $L_{x}$ together with the corresponding fitting function $\kappa=c L_{x}^{\beta}$ with $\beta=0.49 \pm 0.02$. As previously pointed out, the resulting $\beta$ value indicates an anomalous behavior (more specifically, superdiffusive) [17]. Similar $\beta$ values were previously determined by NEMD simulations for different polymeric species [11]. However, we remark that all previous investigations have been addressed to systems containing no more than $10^{4}$ atoms: 
therefore size effects are indeed expected [21]. The present results are obtained for systems containing as many as $\sim 10^{5}$ atoms; this feature guarantees full convergence in the $\beta$ values here reported.

In order to understand whether such an anomalous behavior is affected by dimensionality, we performed the same AEMD simulations on a PEDOT crystal with $0.0789 \leqslant L_{x} \leqslant$ $0.789 \mu \mathrm{m}$, obtained according to the procedure described in [25] and containing up to $624 \times 10^{3}$ atoms. As shown in Fig. 2, the thermal conductivity of the PEDOT crystal shows a dramatic reduction with respect to single chains, resulting in an almost constant for $L_{x} \geqslant 0.5 \mu \mathrm{m}(\beta \sim 0)$. This result confirms that the dimensionality rules over heat conduction. Consistently with Ref. [13], we argue that the unlike behavior of 1D and 3D PEDOT systems can be attributed to the occurrence of strong anharmonic interactions between chains which provide an increased scattering among heat carriers.

The Green-Kubo integral and, therefore, the corresponding $\kappa$ value was calculated by EMD in a single PEDOT chain with $N_{m}=1000$, previously equilibrated for $100 \mathrm{ps}$ at $T=300 \mathrm{~K}$. The autocorrelation function of the heat current vector $\langle J(t) J(0)\rangle$ was then sampled during a 4-ns-long microcanonical simulation. The maximum value chosen for the correlation time was $0.7 \mathrm{~ns}$. In order to improve the statistics, we considered ten different trajectories where different initial conditions for the atomic velocity distributions were imposed. As expected, the only nonzero component of the integral in Eq. (2) was corresponding to the chain direction. Depending on the assigned initial velocities, we observed that the GreenKubo integral differently evolved in time: therefore, for some trajectories (actually 6 out of 10) the Green-Kubo integral was eventually found to reach a constant value, while for the remaining ones we observe an overall divergence. The diverging behavior and its chaotic dependence on the initial conditions was previously observed in different single polymer chains [12-14]. A similar behavior has been also observed in 2D graphene subjected to a uniaxial strain [35]. The chaotic behavior has been identified in the seminal Fermi-PastaUlam paper [36]. Mode-coupling theory [37] attributed such anomalous behavior to the presence of long tails in the heat current correlation function and extremely slow decay toward equipartition, a feature that we have in fact found in our present calculations.

As suggested by Lepri et al. [20], such a slow decay gives rise to the power law provided in Eq. (3) and, correspondingly, to the divergence in $\kappa$. In order to test this hypothesis we calculated the overall thermal conductivity from Eq. (2) by first averaging the heat flux autocorrelation function over all ten trajectories and then by performing the time integration. Accordingly, we fitted $\kappa$ by Eq. (4) (see Fig. 3 red line) obtaining $\delta=0.497 \pm 0.002$. Interesting enough, from the present MD simulations, it results in $\delta=\beta$ as predicted by theory [17]. According to the results so far obtained, the superdiffusive regime is again found for the PEDOT single extended chain. Also in this case, we compared 1D vs 3D PEDOT: Fig. 3 shows $\kappa$ vs $t$ (blue line) which is clearly convergent to a value very close to the one previously calculated by AEMD (dashed blue line).

Finally, we investigated the broadening in time $\sigma^{2}(t)$ of a local energy perturbation. In order to estimate $\sigma^{2}(t)$, we considered a single PEDOT chain with $N_{m}=1000$ previously

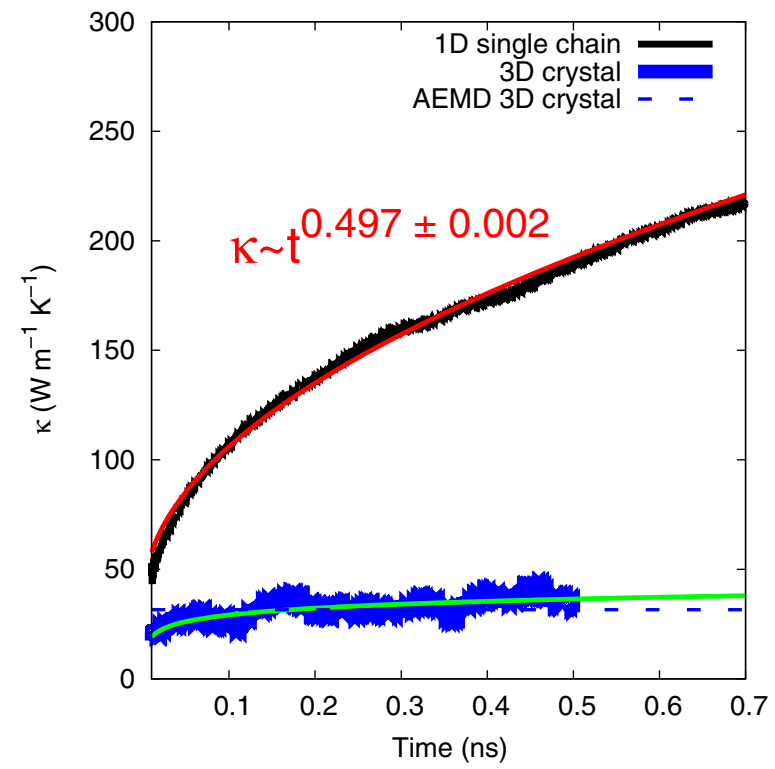

FIG. 3. Black (blue) line: $\kappa$ calculated for a single PEDOT chain (a 3D PEDOT crystal) with $N_{m}=1000$. Full lines (red and green line, respectively) correspond to Eq. (4). The blue dashed line indicates the thermal conductivity computed using AEMD for a 3D PEDOT crystal.

thermalized to an equilibrium state with temperature $T_{0}=$ $300 \mathrm{~K}$ (corresponding to $E_{0}$ ) for $100 \mathrm{ps}$. We mimicked a local energy perturbation by thermostatting the central part of the chain (corresponding to 100 monomers) to a target temperature $T_{1}=500 \mathrm{~K}$ (corresponding to an energy $E_{1}$ ) for $100 \mathrm{ps}$. Then, we aged the system in a microcanonical run eventually estimating the time evolution given in Eq. (5). To suppress statistical fluctuations, an average of over 500 realizations was performed. In Fig. 4 (black line) we show $\sigma^{2}(t)$ before the

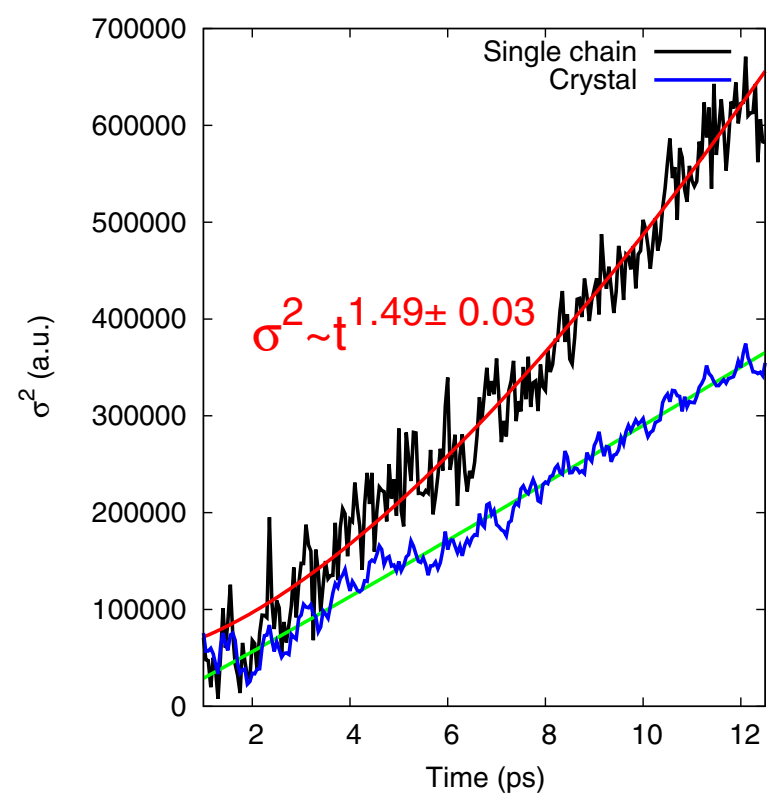

FIG. 4. Time evolution of the broadening of a local energy perturbation $\sigma^{2}(t)$ [see Eq. (5)] in a single 1D PEDOT chain (black line) and a 3D PEDOT crystal (blue line). The red and green lines represent the fitting by Eq. (6). 
occurrence of any turning boundary reflection. By fitting $\sigma(t)^{2}$ by Eq. (6) (see Fig. 4, red line), we obtain $\alpha=1.49 \pm 0.03$ which very accurately fulfills the $\beta=\alpha-1$ relation provided by theory. This result further corroborates the conclusion that thermal transport in single extended PEDOT chains is characterized by a superdiffusive behavior. Also in this case, we perform the same simulation for a 3D PEDOT crystal. Figure 4 (blue line) shows the corresponding time evolution of $\sigma^{2}(t)$ which clearly shows a quasidiffusive behavior with $\alpha=1.04 \pm 0.03$; once again this confirms that the increase of PEDOT dimensionality is able to suppress anomalous thermal transport.

\section{CONCLUSION}

In conclusion, we developed a computational procedure able to supply a thorough characterization of anomalous thermal transport in single PEDOT chains by assessing the occurrence of three scaling laws with unlike physical meaning and showing consistent critical exponents, i.e., highlighting the universality class for different phenomena related to heat transport. We performed a systematic comparison between heat transport in single 1D PEDOT chains vs perfect 3D PEDOT crystals showing that PEDOT single chains exhibit superdiffusive thermal transport while, by increasing the dimensionality to 3D PEDOT crystals, all the anomalies are completely suppressed. On top of providing robust evidence about the actual anomalous superdiffusive character of thermal transport in 1D PEDOT chains, the estimated values for the critical exponent show that thermal transport for this system belong to a novel universality class of superdiffusion characterized by $\beta=$ $1 / 2$. The existence of this regime has never been proven so far neither by experiments nor by atomistic MD simulations based on realistic force fields to describe the interatomic interactions. In this perspective, the class I AMBER model potential used in this work represents an accurate force field for the description of conjugated polymers being its functional form composed by bonding terms (bonds, angles, and dihedrals) as well as nonbonding contributions (Coulomb and van der Waals).

Another important aspect of the present result, is strictly related to the actual functional form of the model potential used to describe the interatomic interactions. It has been proposed that the value of $\beta=1 / 2$ should occur for interatomic potentials that are symmetric (even) when expanded about their minimum. However, in this case the AMBER model potential used for PEDOT is definitely nonsymmetric with respect to the equilibrium position. This unexpected feature stands for the occurrence of superdiffusion characterized by $\beta=1 / 2$ also in force fields that are strictly nonsymmetric with respect to the equilibrium positions.

\section{ACKNOWLEDGMENTS}

Work supported by "Progetto biennale di Ateneo" UniCa/FdS/RAS "Multiphysics theoretical approach to thermoelectricity". We ackowledge as well computational support to CINECA under project ISCRA-IESPR.
[1] R. E. Peierls, Quantum Theory of Solids (Clarendon, Oxford, 1955).

[2] J. M. Ziman, Electrons and Phonons: The Theory of Transport Phenomena in Solids (Oxford University Press, Oxford, 1960).

[3] C. W. Chang, D. Okawa, H. Garcia, A. Majumdar, and A. Zettl, Breakdown of Fourier Law in Nanotube Thermal Conductors, Phys. Rev. Lett. 101, 075903 (2008).

[4] C. W. Chang, A. M. Fennimore, A. Afanasiev, D. Okawa, T. Ikuno, H. Garcia, D. Li, A. Majumdar, and A. Zettl, Isotope Effect on the Thermal Conductivity of Boron Nitride Nanotubes, Phys. Rev. Lett. 97, 085901 (2006).

[5] T. K. Hsiao, H. K. Chang, S. C. Liou, M. W. Chu, S. C. Lee, and C. W. Chang, Observation of room-temperature ballistic thermal conduction persisting over $8.3 \mu \mathrm{m}$ in $\mathrm{SiGe}$ nanowires, Nat. Nanotechnol. 8, 534 (2013).

[6] K. Aoki and D. Kusnezov, Fermi-Pasta-Ulam $\beta$ Model: Boundary Jumps, Fourier's Law, and Scaling, Phys. Rev. Lett. 86, 4029 (2001).

[7] T. Hatano, Heat conduction in the diatomic Toda lattice revisited, Phys. Rev. E 59, R1 (1999).

[8] N. Mingo and D. A. Broido, Carbon Nanotube Ballistic Thermal Conductance and its Limits, Phys. Rev. Lett. 95, 096105 (2005).

[9] G. Zhang and B. Li, Anomalous vibrational energy diffusion in carbon nanotubes, J. Chem Phys. 123, 014705 (2005).

[10] N. Yang, G. Zhang, and B. Li, Violation of Fourier's law and anomalous heat diffusion in silicon nanowires, Nano Today $\mathbf{5}$, 85 (2010).
[11] J. Liu and R. Yang, Length-dependent thermal conductivity of single extended polymer chains, Phys. Rev. B 86, 104307 (2012).

[12] A. Henry and G. Chen, High Thermal Conductivity of Single Polyethylene Chains Using Molecular Dynamics Simulations, Phys. Rev. Lett. 101, 235502 (2008).

[13] A. Henry, G. Chen, S. J. Plimpton, and A. Thompson, 1Dto-3D transition of phonon heat conduction in polyethylene using molecular dynamics simulations, Phys. Rev. B 82, 144308 (2010).

[14] W. Lv, R. M. Winters, F. DeAngelis, G. Weinberg, and A. Henry, Understanding divergent thermal conductivity in single polythiophene chains using Green-Kubo modal analysis and sonification, J. Phys. Chem. A 121, 5586 (2017).

[15] O. Gendelman and A. Savin, Normal heat conductivity in chains capable of dissociation, Europhys. Lett. 106, 34004 (2014).

[16] A. V. Savin and Y. A. Kosevich, Thermal conductivity of molecular chains with asymmetric potentials of pair interactions, Phys. Rev. E 89, 032102 (2014).

[17] S. Lepri, R. Livi, and A. Politi, Thermal Transport in Low Dimensions, Lecture Notes in Physics Vol. 921 (Springer, Berlin, 2016).

[18] B. Li and J. Wang, Anomalous Heat Conduction and Anomalous Diffusion in One-Dimensional Systems, Phys. Rev. Lett. 91, 044301 (2003).

[19] M. H. Ernst, Mode-coupling theory and tails in CA fluids, Physica D (Amsterdam) 47, 198 (1991). 
[20] S. Lepri, R. Livi, and A. Politi, On the anomalous thermal conductivity of one-dimensional lattices, Europhys. Lett. 43, 271 (1998).

[21] S. Lepri, R. Livi, and A. Politi, Universality of anomalous one-dimensional heat conductivity, Phys. Rev. E 68, 067102 (2003).

[22] K. Sun, S.Zhang, P. Li, Y. Xia, X. Zhang, D. Du, F. H. Isikgor, and J. Ouyang, Review on application of PEDOTs and PEDOT:PSS in energy conversion and storage devices, J. Mater. Sci.: Mater. Electron. 26, 4438 (2015).

[23] Q. Wei, M. Mukaida, K. Kirihara, Y. Naitoh, and T. Ishida, Recent progress on PEDOT-based thermoelectric materials, Materials 8, 732 (2015).

[24] G.-H. Kim, L. Shao, K. Zhang, and K. P. Pipe, Engineered doping of organic semiconductors for enhanced thermoelectric efficiency, Nat. Mater. 12, 719 (2013).

[25] C. Genovese, A. Antidormi, R. Dettori, C. Caddeo, A. Mattoni, L. Colombo, and C. Melis, Linking morphology to thermal conductivity in PEDOT: An atomistic investigation, J. Phys. D: Appl. Phys. 50, 494002 (2017).

[26] S. Plimpton, Fast parallel algorithms for short-range molecular dynamics, J. Comput. Phys. 117, 1 (1995); see also the following site: http://lammps.sandia.gov

[27] J. W. Ponder and D. A. Case, Force fields for protein simulations, Adv. Protein Chem. 66, 27 (2003).

[28] J. Wang, R. M. Wolf, J. W. Caldwell, P. A. Kollman, and D. A. Case, Development and testing of a general amber force field, J. Comput. Chem. 25, 1157 (2004).
[29] C. I. Bayly, P. Cieplak, W. Cornell, and P. A. Kollman, A well-behaved electrostatic potential based method using charge restraints for deriving atomic charges: The RESP model, J. Phys. Chem. 97, 10269 (1993).

[30] P. K. Valavala, T. C. Clancy, G. M. Odegard, and T. S. Gates, Nonlinear multiscale modeling of polymer materials, Int. J. Solids Struct. 44, 1161 (2007).

[31] P. E. M. Lopes, O. Guvench, and A. D. MacKerell Jr., Current status of protein force fields for molecular dynamics methods, Methods Mol. Biol. 1215, 47 (2015).

[32] C. Melis, G. Bussi, S. C. R. Lummis, and C. Molteni, Trans-cis switching mechanisms in proline analogues and their relevance for the gating of the 5-HT3 receptor, J. Phys. Chem. B 113, 12148 (2009)

[33] C. Melis, R. Dettori, S. Vandermeulen, and L. Colombo, Calculating thermal conductivity in a transient conduction regime: Theory and implementation, Eur. Phys. J. B 87, 96 (2014).

[34] E. Lampin, P. L. Palla, P. A. Francioso, and F. Cleri, Thermal conductivity from approach-to-equilibrium molecular dynamics, J. Appl. Phys. 114, 033525 (2013).

[35] L. F. C. Pereira and D. Donadio, Divergence of the thermal conductivity in uniaxially strained graphene, Phys. Rev. B 87, 125424 (2013).

[36] E. Fermi, J. Pasta, and S. Ulam, Studies of the nonlinear problems, Los Alamos Scientific Laboratory report LA-1940 (1955).

[37] S. Lepri, Relaxation of classical many-body Hamiltonians in one dimension, Phys. Rev. E 58, 7165 (1998). 